\title{
Uma nova (?) fórmula para o cálculo de rações
}

\author{
A. P. TORRES \\ Escola Superior de Agricultura "Luiz de Queiroz" \\ Universidade de São Paulo
}
INDICE
1) Introdução $\ldots \ldots \ldots \ldots \ldots \ldots \ldots \ldots \ldots$
2) Dedução da fórmula ........... 161
3) Resumo $\ldots \ldots \ldots \ldots \ldots \ldots \ldots \ldots \ldots \ldots 164$
4) Abstract $\ldots \ldots \ldots \ldots \ldots \ldots \ldots \ldots \ldots 164$
5) Bibliografia citada $\ldots \ldots \ldots \ldots \ldots \ldots 165$

(*) Trabalho da Seçăo de Avicultura e Cunicultura 


\section{1) INTRODUÇAOO}

Várias tentativas têm sido feitas por diversos autores no sentido de se conseguir calcular uma fórmula de ração equilibrada, para os animais domésticos, sem o trabalho exaustivo das operações ordinárias.

O próprio Autor já apresentou $(1,2)$ um trabalho neste sentido, bastante satisfatório para a alimentaçáo das aves domésticas, mas, que, por ser baseado no conteúdo em protelna bruta, teria restrita aplicação para os mamiferos domésticos, cujas normas de alimentação se exprimem em proteina digestivel - por enquanto.

Dizemas por enquanto porque, diante das conhecimentos atuais relativos ao aproveitamento do nitrogênio pelos mamiferos, não vemos porque, de um dia para outro, não caiam as antigas e clássicas teorias da diferença de valor nutritivo dos nitrogenados de composiçăo diversa, quando o animal, graças a flora e a fauna microbiana de seu aparelho digestivo, seja capaz de transformá-los.

A regra, por enquanto, é calcularem-se as rações baseando-se na composição em elementos digestíveis da ração, embora já se possa hoje agrupá-los, sem crítica, em princípios nutritivas digestiveis (nutrientes digestiveis) totais e proteina digestivel.

Um método interessante é o divulgado por DUBISKI (3), baseado nas fórmulas de MACIEJEWSKI; neste método estima-se o número de gramas de proteina contidas num quilograma de valor amido de cada alimento, assim como o número de quilos de alimento que produz um quilograma de valor amido. Com êste principio foram organizadas normas, fórmulas e tabelas especials para uso do método.

Entre nós, MOHALYL (4) apresentou um método baseado no que chamiou coeficiente protéico correspondente a quantidade de proteina digestível existente em 100 gramas de valor nutritivo dó álimento e apresenta tabelas e métodos de misturas.

Achamos que éstés métodos poderiam ainda ser mais simplificados, se empregássemas uma fórmula que nas diesse a quantidade de um determinado alimento de uso corrente, para completar as misturas em que entrassem grãos e farelos quaisquer, com o objetivo de equilibrar a ração dentro das normas mais usuais como sejam as de MORRISON (5). 
Dada a simplicidade da deduçáo, extranhamas que até o presente ninguem tenha utilizado tal processo, a menos que não tenha tal fato chegado ao nosso conhecimento.

Poderá este método ser sujeito a críticas que poderão, ou condená-los, ou servir para que passamos aperfeiçó́-lo.

Deixamas de apresentar as tabelas, dispensáveis, no momento, e que preparadas, serão divulgadas no meio daqueles que poderáo utilizá-las: os criadores.

\section{DEDUÇAO DA FORMULA (*)}

\section{Chamemos de}

q a quantidade em que um dos ingredientes da raçăo entra na mistura;

a será o seu teor em proteina digestivel;

b sua riqueza em principios digestiveis totais;

rn sua relaçăo nutritiva;

PD proteina digestivel requerida pelas normas;

NDT nutrientes digestiveis totais exigidos pelas normas;

se procuramas organizar uma ração pelo processo clássico, distribuimos as dados como segue

\begin{tabular}{ccc} 
& P.D. & N.D.T. \\
$\mathbf{q}^{1}$ & $\mathrm{a} 1$ & $\mathrm{~b} 1$ \\
$\mathbf{q}^{2}$ & $\mathrm{a}^{2}$ & $\mathrm{~b} 2$ \\
$\mathbf{q}^{3}$ & $\mathrm{a}^{3}$ & $\mathrm{~b} 3$ \\
$\ldots$ & $\ldots$. & $\ldots$ \\
$\mathbf{q x}$ & $\mathrm{ax}$ & $\mathbf{b x}$ \\
\hline
\end{tabular}

De forma que

$\mathrm{q}^{1} \mathrm{a}^{1}+\mathrm{q}^{2} \mathrm{a}^{2}+\mathrm{q}^{3} \mathrm{a}^{3}+\ldots+\mathrm{q}^{\mathrm{x}}+\mathrm{ax}^{2}=\mathrm{PD}$

e

$q^{1} b_{1}+q^{2} b^{2}+q^{3} b^{3}+\ldots+q \times b x=N D T$

A relaçăo nutritiva $\mathrm{RN}$ exigida pelas normas será

(*) Nas fórmulas constantes dêste artigo os expoentes nada mais significam que indices. 
$\mathrm{RN}=\frac{\mathrm{PD}}{\mathrm{NDT}-\mathrm{PD}}(\mathbf{3})$ e a relaçáo nutritiva rn do alimento $\mathrm{q}^{1}$ $\mathrm{rn}^{1}=\frac{\mathrm{a} 1}{\mathrm{~b}^{1}-\mathrm{a}^{1}}, \mathrm{de} \mathrm{rn}^{2}=\frac{\mathrm{a}^{2}}{\mathrm{~b}^{2}-\mathrm{a}^{2}} \ldots . \mathrm{rnx}=\frac{\mathrm{ax}}{\mathrm{bx}-\mathrm{ax}}=(4) ;$ em (3) porque

$$
\mathrm{NDT}-\mathrm{PD}=\left(\mathrm{b}^{1-a}-\mathrm{a}^{1}\right)+\left(\mathrm{b}^{2}-\mathrm{a}^{2}\right)+\left(\mathrm{b}^{3}-\mathrm{a}^{3}\right) \cdots+\left(\mathrm{bx}^{2}-\mathrm{ax}\right)
$$

podemos escrever que

$$
R N=\frac{q^{1} a_{1}+q^{2} a^{2}+q^{3} a^{3}+\cdots+q \times a x}{q^{1}\left(b^{1}-a^{1}\right)+q^{2}\left(b^{2}-a^{2}\right)+q^{3}\left(b^{3}+a^{3}\right) \ldots+q^{x}\left(b x-a^{x}\right)}
$$

se em (4)

$$
\begin{gathered}
\mathrm{rn} 1=\frac{\mathrm{a} 1}{\mathrm{~b} 1 \div \mathrm{al}} \\
\mathrm{b} 1-\mathrm{a} 1=\frac{\mathrm{a} 1}{\mathrm{rn} 1} \ldots \mathrm{bx}-\mathrm{ax} \frac{\mathrm{ax}}{\mathrm{rnx}}
\end{gathered}
$$

substituindo em (5) temos

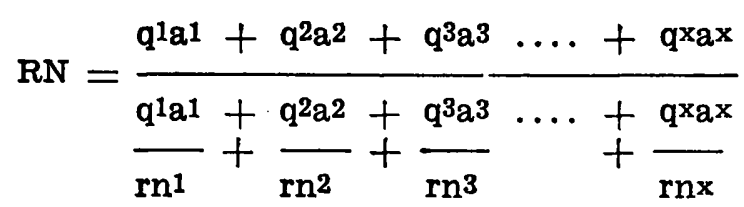

substituindo-se rn pelo seu inverso $\frac{1}{y}$

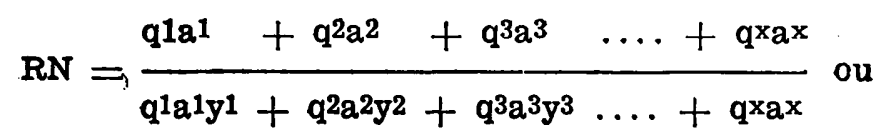


$R N\left(q^{1} a_{1} y^{1}+q^{2} a^{2} y^{2}+\ldots q^{x a x y x}\right)=q^{1} a^{1}+q^{2} a^{2}+\ldots+q \times a x$

O que nos interessa saber é o valor de qx, isto é, uma quantidade de um alimento que dé para o total uma relação nutritiva RN exigida pelas normas.
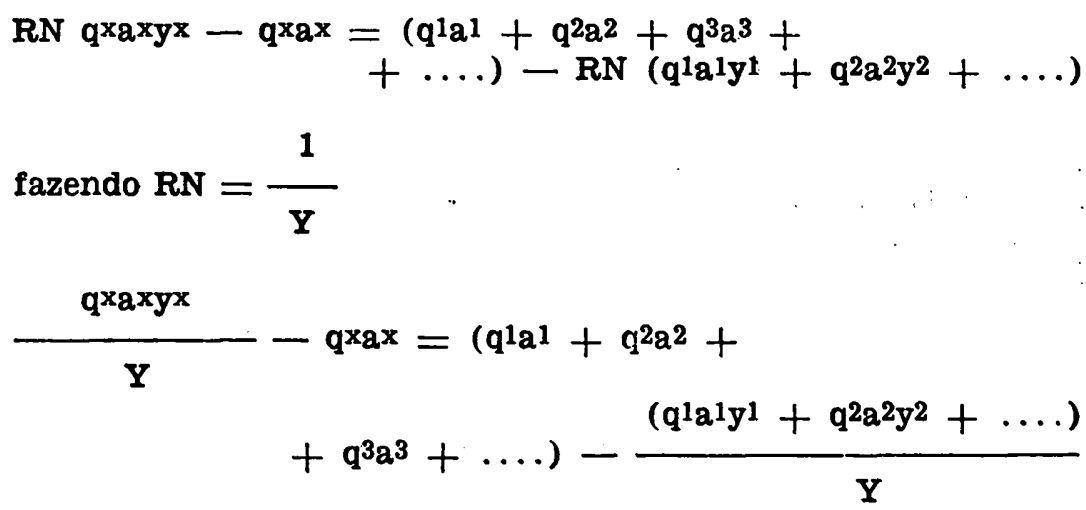
$q \times\left(\frac{a x y x}{Y}-a x\right) \Rightarrow\left(q^{1} a_{1}+q^{2} q^{2}+\right.$ $\left.\mathrm{q}^{3} \mathrm{a}^{3}+\ldots\right)-\frac{\left(\mathrm{q}^{1} \mathrm{a}^{1} \mathrm{y}^{1}+\mathrm{q}^{2} \mathrm{a}^{2} \mathrm{y}^{2}+\ldots\right)}{\mathrm{Y}}$ $\left(q^{1} a^{1}+q^{2} a^{2}+q^{3} a^{3}+\ldots\right)-\frac{\left(q^{1} a^{1} y^{1}+q^{2} a^{2} y^{2}+\ldots\right)}{Y}$ $q x=\frac{\operatorname{axyx}-a x}{Y}$ Multiplicando-se ambos as termas da fração por $\mathrm{Y}$ teremos $q x=\frac{Y\left(q^{1} a^{1}+q^{2} a^{2}+q^{3} a^{3}+\ldots\right)-\left(q^{1} a^{1} y^{1}+q^{2} a^{2} y^{2}+\ldots\right)}{a x y x-Y a x}$ $q^{x}=\frac{Y\left(q^{1} a^{1}+q^{2} a^{2}+q^{3} a^{3}+\ldots\right)-\left(q^{1} a^{1} y^{1}+q^{2} a^{2} y^{2}+\ldots\right)}{a^{x}(y x-Y)}$ 
quando $\mathrm{yx}-\mathrm{Y}$ for negativo năo é passível o balanço desejado.

$$
\mathrm{q}^{\mathrm{x}}=\frac{\sum_{\mathrm{q}^{\mathrm{nan}}}^{\mathrm{q}^{1} \mathrm{a}^{1}}-\sum_{\mathrm{q}^{\mathrm{nanyn}}}^{\mathrm{q}^{1 \mathrm{a} 1 \mathrm{y} 1}}}{\left.\mathrm{ax}^{\mathrm{yx}}-\mathrm{Y}\right)}=\frac{\mathrm{Y} \text { Sqa }- \text { Sqay }}{\mathrm{ax}(\mathrm{yx}-\mathrm{Y})}
$$

Os valores $\mathrm{Y}$, qa, qay, serăo dados pelas nossas tabelas em organizaçăo.

$\mathrm{Na}$ fórmula, a quantidade (q) de um alimento (x), a ser adicionada a uma mistura de alimentas para balanceá-la, afim de alcançar uma relação nutritiva $1: Y$, é igual ao denominador da relação nutritiva desejada (Y), multiplicado pela soma (s) das quantidades de alimento (q) multiplicadas pelo teor de proteina (a) correspondente, menos a soma (s) do produto das quantidades pelo teor de proteina (a), e pelo inverso da relação nutritiva de cada alimento (y); tudo isso dividido pelo teor de proteina (a) do alimento que deve completar a ração, multiplicado pelo diferença entre o inverso da relação nutritiva dêsse alimento e o inverso da relação desejada.

\section{RESUMO}

Visando a obtenção dum método fácil e rápido para calcular rações para animais, o Autor deduz e apresenta uma formula que dará a quantidade dum alimento a ser acrescentada a uma mistura para balanceá-la.

Sugere a necessidade de tabelas apropriadas para facilitar o uso da formula.

\section{ABSTRACT}

Looking for a short and quick method for calculating animal ration, the Author deduces and presents a formula which gives the amount of a particular food to be added to the mixture for a balanced diet.

The necessity of proper tables to facilitate the use of the formula is pointed out. 


\section{BIBLIOGRAFIA CITADA}

1) TORRES, A. DI PARAVICINI, 1946 - Um método prático de racionamento das aves domésticas, in Anais da Esc. Sup. de Agric. "Luiz de Queiroz", 3:313 - 322. Piracicaba.

2) TORRES, A. DI PARAVICINI, 1946 - Um método prático de racionamento das aves domésticas, in Anais da Esc. Sup. de Agric. "Luiz de Queiroz", Nota Suplementar, 3: 323-329. Piracicaba.

3) DUBISKI, J. 1933 - Novo método para determinar a ração alimentar - Resumo in Revue Internacionale d'agriculture, avril, 1933 - Reproduzido in Revista de Agricultura 8: 241--243, Piracicaba.

4) MOHALYL, GABRIEL, 1931 - Tabelas práticas para o cálculo de rações dos Animais Domésticos, in Boletim de Agricultura, 32: 222 - 252, São Paulo.

5) MORRISON, F. B., 1943 - Feeds and Feeding, 20. ed. unabr., Ithaca. 


\section{.}

